\title{
The pattern of urologic care among traumatic spinal cord injured patients
}

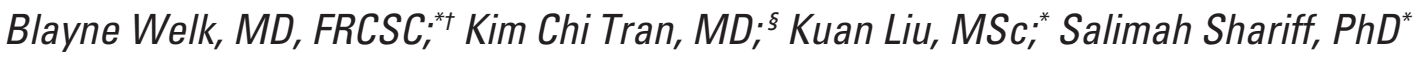

*Institute for Clinical Evaluative Sciences - Western University (ICES Western), London, ON; †Department of Surgery, and Epidemiology and Biostatistics, Western University, London, ON; §Department of Surgery, Western University, London, ON

Cite as: Can Urol Assoc J 2014;8(11-12):e805-9. http://dx.doi.org/10.5489/cuaj.2403 Published online November 24, 2014.

\section{Abstract}

Introduction: We assessed the urologic care patterns of traumatic spinal cord injury (TSCl) patients.

Methods: This was a retrospective cohort study of adult TSCI patients injured between 2002 and 2012. The primary outcome was urologic consultation. The primary exposure was the year of injury. Measured covariates included lesion level, age, gender, comorbidity burden, and socioeconomic status.

Results: We identified 1551 incident TSCI patients who were discharged from a rehabilitation hospital in Ontario between 2002 and 2012. The median follow-up time of this cohort was 5.0 (interquartile range [IQR] 2.9-7.5) years. Within this cohort, 74\% were male, and the mean age was 48 (IQR 33-63) years. In total, 66\% of patients (1022/1551) were seen by a urologist in a median of 0.7 (IQR 0.2-3.0) years after the SCI. Over the study period, there was no change in the proportion of TSCl patients being assessed by a urologist within 1 year of their initial injury (median $55.1 \%$, $p=0.92$ for the trend). An adjusted Cox proportional hazards model demonstrated that TSCI patients who were female (hazard ratio $[\mathrm{HR}] 0.77,95 \%$ confidence interval $[\mathrm{Cl}]$ 0.66-0.92) or over 65 years of age (HR $0.70,95 \% \mathrm{Cl} 0.57-0.85)$ were significantly less likely to be referred to a urologist.

Conclusions: Urologists are often not involved in the care of TSCI patients, and this has not changed significantly over the last 10 years. Females and older patients are significantly less likely to be referred to a urologist.

\section{Introduction}

In Canada there are an estimated 1800 new traumatic spinal cord injuries (TSCI) each year. ${ }^{1}$ These patients have significantly higher rates of healthcare service utilization throughout their lifetime. ${ }^{2}$ Hospital visits are highest in the first year following their injury, and this number remains high 6 years post-injury. ${ }^{3}$ This increased hospitalization rate has a significant direct impact on healthcare costs, and an indirect societal cost in the form of decreased productivity, employment and independence for TSCl patients. ${ }^{4}$ The reason that TSCI patients need to seek emergency medical attention is often due to preventable secondary health conditions related to their TSCl, such as bladder related dysfunction. ${ }^{3,5,6}$

There are only a few consensus guidelines on the management of TSCl patients, and most suggest at least a yearly urologic assessment. ${ }^{7,8}$ A systematic review identified evidence for yearly renal ultrasounds; however the remainder of the urologic follow-up is undefined and based on expert opinion..$^{9-11}$ The actual level of involvement of urologists in the management of TSCl is unknown.

In this study, we examined the current level of urologic involvement among TSCI patients in Ontario, Canada, and assessed if this pattern of care has changed over a 10-year period (2002-2012). Our secondary objective was to assess patient factors associated with receiving urologic care. Methods

\section{Study design}

This was is a retrospective cohort study. Patients were drawn from administrative data from the province of Ontario, Canada (which has a population of about 13 million people who all use a single, publically funded, universal healthcare system). We obtained institutional ethics approval for this study.

\section{Data sources and validity}

Since 2002, the National Rehabilitation System (NRS) has required standardized patient level reporting from all rehabilitation centers in Ontario. All Ontario hospitals are required to submit hospitalization details for patients to the Canadian Institutes for Health Information Discharge 
Welk et al.

Abstract Database. Ontario Health Insurance Plan (OHIP) fee claims are expected to have a high sensitivity and positive predictive value. Data quality has been previously reported. ${ }^{12}$

\section{Population, exposure and outcome definitions}

Our patient cohort of TSCI patients was identified using a previously validated administrative data algorithm based on NRS data (sensitivity 92\%, specificity $97 \%$ ). ${ }^{12}$ We included all adult patients admitted to a rehabilitation facility in Ontario between April 1, 2002 and March 31, 2012 following an incident TSCl. Patients who did not reside in Ontario, those with less than 1 year of follow-up, and those who had a urology visit in the year prior to the date of their injury were excluded (to maximize our measurement of urologist assessments specifically for TSCI related issues, as opposed to routine follow-up of a preexisting condition).

Our primary outcome was first clinical assessment of a $\mathrm{TSCl}$ patient by a urologist; this was determined by using all OHIP fee claim codes which may be used by urologists when assessing a patient. OHIP fee codes were available until March 31, 2013 to ensure all patients had at least a 1 -year follow-up window. For our primary objective, the exposure was year of TSCl.

For our secondary objective, the following variables were included: age, gender, comorbidity index (using the sum of the Aggregated Diagnostic Groups (ADG) from the validated Johns Hopkins University Adjusted Case Groups case-mix system, dichotomized into 0-11 (low comorbidity) versus $\geq 12$ (high comorbidity), ${ }^{13}$ and socioeconomic status (using the Ontario Marginalization Index scores of residential instability, material deprivation, and ethnic concentration as a proxy for individual marginalization); ${ }^{14}$ quintiles were ranked from 1 (least marginalization) to 5 (most marginalization). A planned subgroup analysis was carried out on patients who had lesion level data based on the NRS rehabilitation coding groups (RCG) codes, which allow lesion levels to be dichotomized into quadriplegia (cervical lesions) and paraplegia (thoracic or lumbar lesions) with acceptable accuracy. ${ }^{12}$

\section{Statistical analysis}

Continuous variables were reported as medians with interquartile ranges (IQR). Categorical variables were reported as percentages (proportion). Patients were considered "at risk" for the primary outcome between the injury date to the date of censoring; patients were censored at death, when they left the province, or the end of the follow-up period (March 31, 2013).

For our primary objective, the Cochran-Armitage test for trend was used. A Cox proportional hazards model was used to assess the impact of our secondary exposures on the likelihood of urologist assessment. Log-binomial regression was used for the subgroup analysis of patients with at least a 3-year survival and lesion level data available. Hazard ratios (HRs), relative risk (RR), 95\% confidence intervals $(\mathrm{Cl})$ and $p$ values were reported. SAS 9.3 (SAS Institute, Cary, NC, USA) was used.

\section{Results}

We identified a total of $1551 \mathrm{TSCl}$ patients who met our inclusion criteria (Table 1). A median of 161 patients (IQR 140-169) were identified within each fiscal year from 2002 to 2012. This cohort had a median follow-up of 5.0 (IQR 2.9-7.5) years.

Over our entire study period, $66 \%(1022 / 1551)$ of TSCI patients were assessed at least once by a urologist. The most common referring physician was the family physician (36\%), followed by physiatry $(27 \%)$, and orthopedics/neurosurgery $(23 \%)$. Very few were referred directly from an emergency room physician for their first urologic assessment (3\%). For those who were referred and seen, the median time between $\mathrm{TSCl}$ injury and first urologic assessment was 0.7 (IQR 0.23.0) years. Once a patient was seen by a urologist, they had a median of 3 (IQR 1-7) subsequent visits during the study period (for a median rate of 0.8 visits per patient year of follow-up). Eleven urologists in the province saw $70 \%$ of these patients (750/1065).

Across each fiscal year from 2002 to 2012, the median proportion of patients seen by a urologist for the first time within 1 year of their injury was 55.1\% (IQR 54.3-55.8\%). There was no significant linear trend over this 10 -year period $(p=0.92)$. Similarly, the median proportion of patients seen by a urologist within 2 years of their injury $(62.0 \%$,

\section{Table 1. Cohort demographics}

\begin{tabular}{|c|c|}
\hline & Entire cohort $(n=1551)$ \\
\hline Gender (male) & $74 \%(1144)$ \\
\hline $\begin{array}{l}\text { Median age in years at time of } \mathrm{TSCl} \\
\text { (IQR) }\end{array}$ & $48(33-63)$ \\
\hline \multicolumn{2}{|l|}{ ADG Comorbidity score } \\
\hline $0-11$ & $77 \%$ \\
\hline$\geq 12$ & $23 \%$ \\
\hline \multicolumn{2}{|l|}{$\begin{array}{l}\text { Ontario Marginalization scores } \\
\text { quintiles }(\mathbf{Q} 1, \mathbf{Q}, \mathbf{Q} 5)^{*}\end{array}$} \\
\hline Residential instability & Q1 20\%, Q3 15\%, Q5 16\% \\
\hline Material deprivation & Q1 18\%, Q3 19\%, Q5 14\% \\
\hline Ethnic concentration & Q1 13\%, Q3 15\%, Q5 24\% \\
\hline \multicolumn{2}{|l|}{ Level of SCI } \\
\hline Quadriplegia (Cervical lesion) & $37 \%(573)$ \\
\hline Paraplegia (Thoracic/lumbar lesion) & $43 \%(663)$ \\
\hline Missing RCG code & $20 \%(315)$ \\
\hline $\begin{array}{l}\text { *For the Ontario Marginalization score, Q1 is consi } \\
\text { SES group. TSCl: traumatic spinal cord injury; SCl: } \\
\text { coding groups. }\end{array}$ & $\begin{array}{l}\text { d highest SES, and } \mathrm{Q} 5 \text { is the lowest } \\
\text { al cord injury; RCG: rehabilitation }\end{array}$ \\
\hline
\end{tabular}




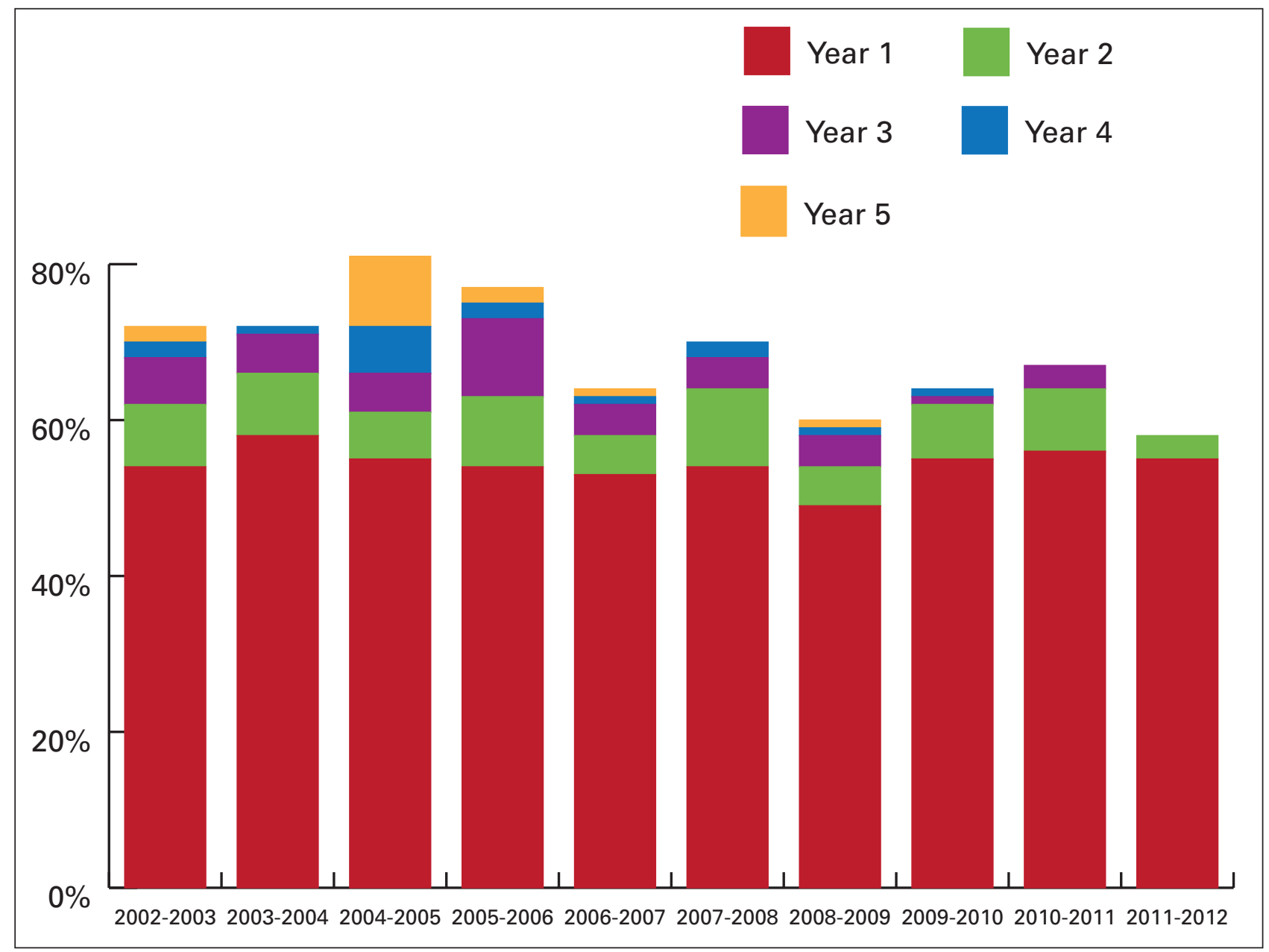

Fig. 1. Proportion of traumatic spinal cord injury (TSCI) patients referred to urology over time, based on the year of injury. Each stacked shaded bar represents the additional proportion of patients with a TSCI occurring during the specified fiscal year that was referred to a urologist. Different shading accounts for the number of additional years after TSCI that the referral was made. Because of a maximum of follow-up time of March 31, 2013, not all fiscal periods have complete year 2 to 5 follow-ups.

IQR 59.8-63.8\%) did not change significantly over time $(p=0.44)$ (Fig. 1).

A Cox proportional hazards model was used to assess the significance of age, gender, comorbidities, and socioeconomic status on the likelihood of a urologic assessment (Table 2). Female patients (HR 0.77, 95\% Cl 0.66-0.92), and patients over 65 (HR 0.70,95\% Cl 0.57-0.85) were significantly less likely to be seen by a urologist after TSCl. Among the subgroup of 946 patients with at least a 3-year follow-up after TSC, and with lesion level information (quadriplegia compared to paraplegia), the lesion level was not a significant contributor to early (within 1 year) urologic referral (RR 0.96, 95\% Cl 0.88-1.04, $p=0.30$ ).

\section{Discussion}

This study is the first to examine the TSCI patient's pattern of urologic care. We demonstrated that only $55 \%$ of patients saw a urologist within 1 year of their injury, and very few patients were referred in subsequent years. This suggests that the momentum for urologic referral is centered on the initial rehabilitation period. This may be because of the frequent contact with the rehabilitation team, and the ease at which patients can discuss bladder-related concerns. It is also likely that many of these patients had obvious urologic issues which necessitated a urologic referral.

We conceptualized the process of urologic care for a TSCI patients as follows: (1) the patient is referred to a urologist by a physician; (2) the urologist must accept the referral; (3) the patient must attend the appointment with the urologist; (4) the urologist must offer appropriate investigations and treatment; and (5) the patient must follow through with the recommended investigations and accept the proposed treatments. Any disruption to one of these steps, or a failure to transition from one step to another potentially interrupts the delivery of optimal urologic care. If patients cannot reliably 
Welk et al.

\begin{tabular}{|c|c|c|}
\hline & HR (95\% Cl) & $p$ value \\
\hline Gender (reference male) & $0.77(0.66-0.92)$ & 0.0035 \\
\hline \multicolumn{3}{|l|}{ Age (reference $18-39$ years) } \\
\hline $40-64$ years & $0.94(0.81-1.09)$ & 0.4042 \\
\hline$\geq 65$ & $0.70(0.57-0.85)$ & 0.0005 \\
\hline $\begin{array}{l}\text { ADG comorbidity score (reference } \\
{[0-11 \text {, low comorbidity]) }}\end{array}$ & $0.98(0.82-1.16)$ & 0.7726 \\
\hline \multicolumn{3}{|l|}{ Ontario Marginalization scores } \\
\hline Residential instability & $0.99(0.94-1.05)$ & 0.7777 \\
\hline Material deprivation & $0.99(0.94-1.05)$ & 0.7160 \\
\hline Ethnic concentration & $1.03(0.98-1.08)$ & 0.2683 \\
\hline
\end{tabular}

Socioeconomic status was determined using the Ontario Marginalization scores (which were used as continuous variables). TSCl: traumatic spinal cord injury; HR: hazard ratio; $\mathrm{Cl}$ : confidence interval; ADG: aggregated diagnostic groups.

transition through the steps in this pathway, then clinical practice guidelines on recommended urologic interventions and screening cannot be fully implemented. Efforts need to be focused on potential barriers in the above pathway to ensure the maximal positive impact is realized. While completion of this care partway may seem straightforward, there are multiple factors that can impede a TSCI patient's access to medical care. ${ }^{5}$

Our study addressed the question of how many patients transition through steps 1 to 3 of the above pathway. There are a number of potential reasons why a larger proportion of $\mathrm{TSCl}$ do not see a urologist. Firstly, alternative care providers (such as physiatrists) may be taking over the urologic care for some patients. While this is certainly appropriate for many urologic issues, there are specific investigations (such as cystoscopy) and specific interventions (such as intravesical onabotulinum toxin) that require a urologist. Secondly, due to these patients' complex urologic issues, not all urologists may accept elective referrals of TSCl. This may hinder physiatrists and general practitioners in their attempts to access urologic care. Indeed, we found that most TSCI patient care was being provided by a small group of urologists, which suggests that a subspecialty interest in this area is likely an important factor for a TSCl patient trying to access urologic care. Thirdly, patients may not attend clinic appointments due their frustrations with the medical system, preference for specialized expertise, and physical accessibility issues. ${ }^{5,15}$

Finally, there may be a perception that TSCI patients only need urologic assessment in certain situations. The vast majority of patients, especially those in this cohort who all underwent significant inpatient rehabilitation, will need some type of urinary device for voiding. ${ }^{16}$ While there are obvious situations that would mandate a urology consult, there are also important potential screening tests and preventive counselling that urologists are well-suited to offer. There are demonstrated advantages in terms of quality of life ${ }^{17}$ and complication rates ${ }^{18}$ with certain methods of bladder management - for these issues, a discussion with a urologist may help patients make a fully informed decision about the investigation and management of their bladder.

We demonstrated that female patients with a TSCI were significantly less likely to be referred to a urologist compared to male patients. This is paradoxical given the importance female $\mathrm{TSCl}$ patients place on their bladder function ${ }^{19}$ and the high frequency at which they undergo urologic procedures. ${ }^{20}$ Reasons for this may be related to a tendency in patients to manage their bladder with an indwelling urethral catheter. The assumption could then be that the bladder is "treated," and no further urologic intervention or consultation is necessary despite the potential long-term complications associated with permanent catheterization. Similarly, the greater functional impairment of older patients post-TSCI versus younger patients ${ }^{21}$ and a preference for an indwelling catheter may account for the reduced urologic referral among patients $>65$ years of age.

Current guidelines for the urologic management of TSCI patients are vague $^{8}$ ("generally a urologic evaluation is done every year") or based on an investigation schedule selected by experts with little evidentiary basis to support the associated healthcare costs and patient inconvenience, and little guidance on how to interpret abnormal results in the setting of an asymptomatic patient. ${ }^{7}$ In a sampling of Canadian urologists, $80 \%$ stated that they routinely use renal ultrasound and urodynamics to follow neurogenic bladder patients. ${ }^{11}$ Similar results were obtained from a survey of American members of the Society for Urodynamics and Female Urology. ${ }^{10}$ While these surveys provide an evaluation of the attitudes and optimal practice patterns of urologists, they do not measure actual performance or quantify patients who are never referred to a urologist for management. This study will be important for future evidence-based guidelines and selection of quality of care indicators.

Limitations of this study include the use of administrative data, which provided a large and comprehensive patient sample, but with limited clinical details (such as the exact lesion level, functional impairment, or method of bladder management). We were unable to ascertain the reason for urologic assessment, and although this study addressed referral for urologic care, it did not measure the quality of such care.

\section{Conclusion}

A significant proportion of TSCI patients do not see a urologist. Female patients and patients over 65 are less likely to be referred to a urologist. The impact of this practice pattern on the urologic health of TSCI patients requires further study.

Acknowledgements: Funding provided by the Ontario Neurotrauma Foundation, in partnership with the Rick Hansen Institute. This study was supported by the Institute for Clinical Evaluative Sciences 
(ICES), which is funded by an annual grant from the Ontario Ministry of Health and Long-Term Care (MOHLTC). The study was conducted through ICES Western which is funded by an operating grant from the Academic Medical Organization of Southwestern Ontario (AMOSO). The opinions, results and conclusions reported in this paper are those of the authors and are independent from the funding sources. No endorsement by ICES, the Ontario MOHLTC or AMOSO is intended or should be inferred.

Competing interests: Dr. Welk, Dr. Tran, Dr. Liu and Dr. Shariff declare no competing financial or personal interests.

This paper has been peer-reviewed.

\section{References}

1. Farry A, Baxter D. The incidence and prevalence of spinal cord injury in Canada. Rick Hansen Institute. Urban Futures Report,2010:1-57.

2. Guilcher SJT, Munce SEP, Couris CM, et al. Health care utilization in non-traumatic and traumatic spinal cord injury: a population-based study. Spinal Cord 2010;48:45-50. http://dx.doi.org/10.1038/sc.2009.78

3. Guilcher SJT, Craven BC, Calzavara A, et al. Is the emergency department an appropriate substitute for primary care for persons with traumatic spinal cord injury? Spinal Cord 2012;51:202-8. http://dx.doi. org/10.1038/sc.2012.123

4. Munce SEP, Wodchis WP, Guilcher SJT, et al. Direct costs of adult traumatic spinal cord injury in Ontario. Spinal Cord 2013;51:64-9. http://dx.doi.org/10.1038/sc.2012.81

5. Guilcher SJT, Craven BC, Lemieux-Charles $L$, et al. Secondary health conditions and spinal cord injury: An uphill battle in the journey of care. Disabil Rehabil 2012:35;894-906. http://dx.doi.org/10.310 9/09638288.2012.721048

6. Hitzig SL, Tonack M, Campbell KA, et al. Secondary health complications in an aging Canadian spinal cord injury sample. Am J Phys Med Rehabil 2008;87:545-55. http://dx.doi.org/10.1097/ PHM.0b013e31817c16d6

7. Stöhrer $M, B l o k B$, Castro-Diaz $D$, et al. EAU guidelines on neurogenic lower urinary tract dysfunction. Eur Urol 2009;56:81-8. http://dx.doi.org/10.1016/i.eururo.2009.04.028
8. Consortium for Spinal Cord Medicine: Bladder management for adults with spinal cord injury: A clinical practice guideline for health-care providers. J Spinal Cord Med 2006;29:527-73.

9. Cameron AP, Rodriguez GM, Schomer KG. Systematic review of urological followup after spinal cord injury. J Urol 2012;187:391-7. http://dx.doi.org/10.1016/i.juro.2011.10.020

10. Razdan S, Leboeuf L, Meinbach DS, et al. Current practice patterns in the urologic surveillance and management of patients with spinal cord injury. Urology 2003;61:893-6. http://dx.doi.org/10.1016/ S0090-4295(02)02518-9

11. Blok BFM, Karsenty G, Corcos J. Urological surveillance and management of patients with neurogenic bladder: Results of a survey among practicing urologists in Canada. Can I Urol 2006;13:3239-43.

12. Welk $B$, Loh E, Shariff SZ, et al. An administrative data algorithm to identify traumatic spinal cord injured patients: A validation study. Spinal Cord 2014;52:34-8. http://dx.doi.org/10.1038/sc.2013.134

13. The Johns Hopkins ACG® Case-Mix System Reference Manual. 7 ed. Baltimore: Health Services Research and Development Center; 2007:1-11.

14. Matheson Fl, Dunn JR, Smith KLW, et al. Development of the Canadian Marginalization Index: A new tool for the study of inequality. Can J Public Health 2012;103:S12-6.

15. McColl MA, Aiken A, McColl A, et al. Primary care of people with spinal cord injury: Scoping review. Can Fam Physician 2012;58:1207-16.

16. Neurogenic Bladder Turkish Research Group, Yıldız N, Akkoç Y, et al. Neurogenic bladder in patients with traumatic spinal cord injury: Treatment and follow-up. Spinal Cord 2014;52:462-7. http://dx.doi. org/10.1038/sc.2014.41

17. Liu C-W, Attar KH, Gall A, et al. The relationship between bladder management and health-related quality of life in patients with spinal cord injury in the UK. Spinal Cord 2010;48:319-24. http://dx.doi. org $/ 10.1038 / s c .2009 .132$

18. Weld KJ, Dmochowski RR: Effect of bladder management on urological complications in spinal cord injured patients. J Urol 2000;163:768-72. http://dx.doi.org/10.1016/S0022-5347(05)67800-7

19. Pentland W, Walker J, Minnes $P$, et al. Women with spinal cord injury and the impact of aging. Spinal Cord 2002;40:374-87. http://dx.doi.org/10.1038/si.sc.3101295

20. Lombardi $G$, Musco $S$, Celso $M$, et al. A retrospective study on female urological surgeries over the 10 years following spinal cord lesion. Spinal Cord 2013;51:688-93. http://dx.doi.org/10.1038/sc.2013.64

21. Furlan JC, Fehlings MG. The impact of age on mortality, impairment, and disability among adults with acute traumatic spinal cord injury. J Neurotrauma 2009;26:1707-17. http://dx.doi.org/10.1089/ neu.2009.0888

Correspondence: Dr. Blayne Welk, B4-667 St Joseph's Health Care, 268 Grosvenor St., London ON N6A 4V2; fax: 519 646-6037; blayne.welk@sihc.london.on.ca 